\title{
Caregivers of family members with dementia described the experience of quality respite as a 3 phase cognitive journey towards a sense of "being free"
}

Strang VR, Haughey M. Respite-a coping strategy for family caregivers. West J Nurs Res 1999 Aug;21:450-71.

QUESTION: How do caregivers of family members with dementia experience respite?

Design

Qualitative interpretive approach.

Setting

Edmonton, Alberta, Canada.

\section{Participants}

10 white caregivers ( 8 wives, 1 husband, and 1 son) who were $47-82$ years of age (mean age $70 \mathrm{y}$ ) were recruited from self help groups, informal community connections, and home care agencies. 9 of the caregivers were retired, and the duration of caregiving ranged from 6 months to 10 years (mean 5 y). 6 of the care recipients had Alzheimer's-type dementia and 4 had multi-infarct dementia associated with stroke.

\section{Methods}

Data saturation determined the number of caregivers interviewed. Each caregiver participated in 2 indepth, loosely structured, 60-90 minute interviews (2 months apart) to discuss their experiences of caregiving and respite. Data analysis procedures included substantive coding, memoing, and integrative diagramming. Credibility was established by 2 other researchers verifying the analytical codes and emerging themes. During the second interview, caregivers verified the authenticity of the analyses by confirming and reinterpreting the researchers' findings.

\section{Main findings}

There emerged an image of 2 spheres of existence: a large, dominating sphere of caregiving and a smaller, but no less special sphere, where they experienced respite. The quintessential meaning of respite was "to be free"-that is, free to be themselves, to pursue their own interests, to not worry about their family member, and to not be in the role of caregiver.

Achieving respite was a 3 phase "cognitive journey": recognising the need to get out of the caregiver world; giving themselves permission to get away from caregiving responsibilities for a while (and realising that such self serving behaviour was appropriate); and finally, realising the availability and adequacy of social supports that would allow them to get away.

Time was another dimension of the respite experience, and involved both the duration and quality of the experience. Short respite intervals (such as when family members attended adult day programmes) were often subsumed into the "ebb and flow of the caregivers' daily lives" and used to run errands or do chores; caregivers rarely considered these to be respite times. Even if caregivers could engage in personal activities during these short respites, the freedom was fleeting as they were still oriented to caregiving responsibilities, such as being home when the family member returned. Longer respite intervals, usually associated with the use of institutional respite services, led to a sense of recuperation or "recharging of batteries". Quality of the respite experience was more important than duration.

\section{Conclusions}

Caregivers of family members with dementia described the respite experience as a separate sphere of their lives where they were "free" from caregiving responsibilities and "free" to be themselves. Experiencing respite involved a "cognitive journey" of recognising the need to get out of the caregiving world, giving themselves permission to get away, and having appropriate social supports to facilitate getting away.

\section{COMMENTARY}

Respite care is believed to reduce the burden of the caregiving situation and to allow families that would otherwise have to resort to nursing home placement to continue care. The findings of studies on the effect of these services on caregivers are inconsistent. ${ }^{1}$ It may be that research based on formal service perspectives is unable to explain how and why caregivers benefit from respite services. To address this gap in understanding, Strang and Haughey conducted in depth interviews with 10 family caregivers of persons with dementia. The quality of the study was high, with the inclusion of appropriate informants. Moreover, the trustworthiness of the data analysis was assured through peer debriefing and member checking.

The lack of theoretical understanding of how respite works has hindered the development of effective respite programmes. This study suggests that caregivers must use internal coping mechanisms to identify their need for respite and to give themselves "permission" to use it. We know that caregivers' perceived need for respite accounts better for patterns of service use than more objective measures, such as the functional status of the care receiver. ${ }^{2}$ Thus, the ability to assess caregiver readiness to accept respite could help providers to identify service needs better. The findings suggest that caregiver comfort with receiving aid is another important clinical dimension as is the need for high quality information about services and how they are provided. Furthermore, the 3 stage nature of the decision processes around respite use means that provider follow up of those caregivers who initially refuse services is important.

The lack of ethnic, educational, and financial diversity in this study sample makes it difficult to generalise the findings to all social groups. Cognitive coping may not be a universal strategy among all caregivers. Further research is needed to explore how the strategies of minority group caregivers may differ from those described in this study.

Deborah Messecar, RN, PhD Assistant Professor, School of Nursing Oregon Health Sciences University, Portland, Oregon, USA

1 Knight BG, Lutzky SM, Macofsky-Urban F. A meta-analytic review of interventions for caregiver distress: recommendations for future research. Gerontologist 1993;33:240-8.

2 Montgomery RJV. Examining respite: promises and limitations. In: RA Kane, JD Penrod, editors. Family caregiving in an aging society. Policy perspectives. Thousand Oaks, CA: Sage, 1995:29-45. 